\title{
VOLUNTAD DE PODER Y TRANSFINITUD: CONVERGENCIA Y COLISIÓN ENTRE NIETZSCHE Y GARCÍA BACCA
}

\author{
XAVIER GIMENO MONFORT \\ Universidad de Valencia
}

\begin{abstract}
RESUMEN: En el presente artículo trataremos un tema inédito dentro de los estudios sobre la vida y obra de García Bacca. En especial, trataremos de ver en qué medida la obra de García Bacca puede ser interpretada como una expansión de los límites que, en opinión de Heidegger, la propuesta metafísica nietzscheana lleva asociado de modo intrínseco. En especial, trataremos de analizar comparativamente ambas propuestas metafísicas con el fin de demostrar que si bien parte de la génesis de la propuesta filosófica garcibacquiana responde parcialmente ciertos elementos característicos de Nietzsche, el progresivo contacto de García Bacca con otras perspectivas políticas, filosóficas, económicas, etc., provocan una aproximación paralela a las obras de Machado y Marx. Dicha aproximación a estos y otros factores de influencia, provocan una transformación y ampliación en su propuesta filosófica.
\end{abstract}

PALABRAS CLAVE: Nietzsche; García Bacca ontología; Nos; expansión; límite.

\section{Will to Power and Transfinity:}

\section{Collision and Convergence between Nietzsche And García Bacca}

\begin{abstract}
In this article we will try an unreleased track in studies on the life and work of García Bacca. In particular, we try d see to what extent the work of Garcia Bacca can be interpreted as an expansion of the limits, according to Heidegger, Nietzsche's metaphysics proposal intrinsically associated leads. In particular, try to comparatively analyze both metaphysical proposals in order to demonstrate that although part of the genesis of philosophical proposal garcibacquiana partially responds certain characteristic economic elements of Nietzsche, progressive García Bacca contact with other political views, philosophical, etc., cause a parallel to the works of Marx and Machado approach. This approach to these and other factors of influence, causing a transformation and expansion in its philosophical proposal.
\end{abstract}

KEY WORDS: Nietzsche; Garcia Bacca; ontology; We; expansión; limit.

\section{INTRODUCCIÓN}

En el presente artículo vamos a tratar de analizar las posibilidades que proporciona la propuesta filosófica garcibacquiana a la hora de considerar una posible expansión a los límites que, en opinión de Heidegger, constituye el acabamiento del paradigma filosófico clásico a manos de Nietzsche. Para ello, analizaremos en primer lugar, el hecho de que la propuesta filosófica garcibacquiana bebe, en cierto sentido, de la propuesta nietzscheana. Trataremos de demostrar que dicha influencia y poso nietzscheano en la propuesta filosófica garcibacquiana no se restringe, ni puede ser reducida únicamente, a una asunción o asimilación sin más por parte de García Bacca de las bases terminológicas y ontológicas de la filosofía nietzscheana. Es más, trataremos de demostrar el hecho peculiar, pero natural en términos garcibacquianos, 
de cómo la propuesta filosófica de García Bacca (al menos en los años 40 del pasado siglo), constituye una simbiosis y convivencia pacífica tanto de las bases metafísicas nietzscheanas por un lado, así como de los fundamentos platónicos a los que García Bacca tanto tiempo dedicara y respetara a lo largo de su labor como filósofo: «Sin duda alguna, a quien debo más es a Platón. Manera de pagarle: traducir sus Obras completas» (García Bacca, 2000 p. 97).

Tras exponer la unión que establece García Bacca entre la propuesta nietzscheana y la platónica, es decir, entre el filósofo como sujeto endemoniado (platón) y la metáfora nietzscheana de la «maroma» como expresión ontológica del equilibrio del filósofo entre el superhombre y el animal, trataremos de definir el punto de encuentro específico entre Nietzsche y García Bacca como filósofos de la creación y la invención. Es decir, como autores que describen un estatus ontológico asociado al estado dionisíaco, creativo, inventivo y dialéctico que, en esencia, identifica al ente como un sujeto que lucha por ir siempre más allá de sí mismo. Para dar cuenta de ello, compararemos y analizaremos las categorías de Voluntad de poder nietzscheana y la transfinitud garcibacquiana.

Establecida dicha relación de base, entraremos en el asunto que tal vez condensa el sentido principal del presente trabajo, a saber, el tipo de colisión que se establece entre ambos autores. Por lo pronto, adelantamos que el tipo de colisión entre ambas propuestas es de tipo «lateral». Es decir, el hecho de que García Bacca asiente parte de sus premisas metafísicas en el esquema ontológico nietzscheano, no salva a la propuesta garcibaccquiana de colisionar lateralmente con Nietzsche. Tal y como trataremos de demostrar, la principal consecuencia de dicho impacto lateral es que, en lo que a García Bacca se refiere, su propuesta filosófica sufre una desviación en su dirección original que, a nuestro juicio, la impulsa a nuevas dimensiones ontológicas y, sobre todo, sociales. La desviación de la que hablamos provoca, a nuestro parecer, que la propuesta garcibacquiana logre cubrir o dar respuestas a cuestiones que, en el caso de Nietzsche son, cuanto menos, actualmente problemáticas desde el orden de discurso de la facticidad social y política.

En resumidas cuentas, trataremos de ver cómo la propuesta garcibacquiana (desde cierto orden de discurso no estrictamente ontológico), logra, decimos, cubrir e incluso ampliar las insuficiencias intrínsecas de la propuesta metafísica nietzscheana. Tal y como defendiera Heidegger, posiblemente la propuesta metafísica nietzscheana constituya el acabamiento del paradigma metafísico clásico.

\section{La simbiosis ontológica garcibacouiana entre Platón y Nietzsche}

No abundan las referencias explícitas realizadas por García Bacca a Nietzsche. No es menos cierto, el hecho de que García Bacca no acostumbraba a realizar referencias bibliográficas en sus obras salvo en contadas ocasiones, 
circunstancias o lugares especiales ${ }^{1}$. Debido a su forma de trabajar, sumado a una cierta distancia con las formas actuales de hacer filosofía respecto a los usos y costumbres establecidos por la academia, dificulta en muchas ocasiones establecer puntos de referencias bibliográficos concretos respecto a temas o autores. En el caso que nos ocupa, es decir, tratar de establecer un punto de referencia bibliográfico-histórico en la obra de García Bacca a partir del cual determinar la influencia de Nietzsche se antoja, cuanto menos, ciertamente complejo.

En lo que a nosotros respecta, consideramos que la primera referencia relativamente explícita y seria que García Bacca hace sobre Nietzsche se encuentra en el prólogo de su obra de 1940 Invitación a filosofar ${ }^{2}$. Allí dice García Bacca: «Toda filosofía viva y en trance vital es dionisíaca; es una borrachera de ideas; y el filósofo, en cuanto tipo de vida, es un Baco, un beodo más sutil y considerado que los vulgares chispos». (García Bacca, 1940, p. 2).

El hecho de que García Bacca relacione los conceptos de «filosofía» y «vida» con el principio dionisíaco es, a nuestro juicio, una referencia a uno de los temas transversales de la filosofía nietzscheana, a saber, la crítica y distanciamiento de la filosofía apolínea surgida en la Grecia clásica. Las palabras de García Bacca tratan de manifestar, cuanto menos, una cierta simpatía y filiación por la defensa de la perspectiva dionisíaca desarrollada por Nietzsche. Una perspectiva que, en contra de lo que determinaba la filosofía socrática y platónica, trata de recuperar y restaurar la importancia de las dimensiones corporales y emocionales del hombre como fuente de conocimiento y de vida. Dice Nietzsche:

"Contra la moral, pues, se levanta entonces, con este libro problemático, mi instinto, como un instinto defensor de la vida, y se inventa una doctrina

1 En una de sus obras más importantes, Metafísica, publicada en 1963, García Bacca sentencia en el prólogo que sus influencias van desde la A de Aristóteles, hasta la Z de Zubiri. En una obra tan importante como Metafísica, García Bacca no cita a ningún autor u obra de referencia, dejando al lector absolutamente desamparado ante la tarea de reconstruir un argumento a partir de sus conocimientos. A pesar de la inclinación garcibacquiana por la ausencia de citas, en el año 2000 la editorial Anthropos publicó su Confesiones. Autobiografía intima y exterior. En la segunda parte de sus Confesiones, García Bacca apunta las que, a su parecer, son los «impactos» de fondo. Es decir, nos revela qué y cuáles son los autores y obras que de un modo determinante, influyeron en su vida y obra. También es de rigor intelectual, citar la obra de 1947 Nueve grandes filósofos contemporáneos y sus temas. En ella, García Bacca realiza un repaso sobre 9 nombres de la filosofía contemporánea en una suerte de homenaje y análisis crítico a partes iguales. Actualmente, sigue siendo un tema de discusión vivo y activo, las críticas recibidas por el conjunto de estudiosos sobre la vida y obra de García Bacca, respecto al modo de trabajo y la ausencia casi permanente de citas y referencias bibliográficas en sus trabajos.

2 García Bacca, J. D., Invitación a filosofar. Vol I: La forma del conocer filosófico, y en: México: Fondo de Cultura Económica, 1940. Nosotros trabajamos con la edición electrónica en: http://www.cervantesvirtual.com/servlet/SirveObras/12482399770132621865846/ p0000001.htm\#1. 
y una valoración radicalmente opuestas de la vida, una doctrina y una valoración puramente artísticas, anticristianas. cómo denominarlas? En cuanto filólogo y hombre de palabras las bauticé, no sin cierta libertad — ¿pues quién conocería el verdadero nombre del Anticristo?- con el nombre de un dios griego: las llamé dionisíacos». (Nietzsche, 2012)

Pero no sólo la referencia de García Bacca a la dimensión dionisíaca es una guiño sin más a la tesis nietzscheana. La referencia garcibacquiana a la vitalidad dionisíaca tiene que ver con otro factor determinante dentro de la vida y la obra del filósofo español. Su obra Invitación a filosofar puede ser considerada, en líneas generales, como la primera obra en la que García Bacca da inicio a una nueva forma de hacer filosofía. Las obras de García Bacca de finales de los años veinte y casi la totalidad de los años treinta responde, esencialmente, a cuestiones de corte teológico o, en otros casos, lógico-científico ${ }^{3}$. Puede decirse pues, que en un sentido profundo, García Bacca hacia finales de los años 30 del pasado siglo está inmerso en un salto dialéctico de lo apolíneo a lo dionisíaco tanto vital, así como filosóficamente hablando.

Que se produzca dicho salto dialéctico en la vida y obra de García Bacca no tiene, necesariamente, que asumirse como un salto definitivo donde lo absorbido dialécticamente hablando, quede olvidado o superado sin más. Nada más lejos de la realidad. Tal y como trataremos de demostrar, en el momento en el que nos encontramos dentro de la gestación ontológica garcibacquiana, el filósofo español está inmerso en un proceso dialéctico de asunción y asimilación de nuevas perspectivas filosóficas (es el caso de la filosofía nietzscheana y heideggeriana) pero, también, es el momento en el que las propuestas filosóficas clásicas como la de Platón, todavía conserva un peso y valor notable dentro de la filosófica garcibacquiana. Por esa razón, tal y como podremos comprobar, García Bacca extrae ciertos principios característicos de la propuesta nietzscheana como ocurre con el impulso de la filosofía dionisíaca que, al mismo tiempo, por muy difícil que pueda resultar de creer, terminará combinando legítimamente con propuestas tan aparentemente opuestas como la platónica.

Por esa razón, la comprensión de la unión llevada a acabo por García Bacca entre Platón y Nietzsche es crucial y compleja al mismo tiempo. En primer lugar, García Bacca se refiere a lo «demoníaco» en los mismos términos que Platón en su obra El Banquete: «Todo demoníaco es algo intermedio entre lo divino y lo mortal» (202e). En segundo lugar, García Bacca toma las definiciones platónicas de los conceptos de «sabio» $\mathrm{y}$ «filósofo» de la citada obra de Platón ${ }^{4}$

\footnotetext{
3 Son muchas las referencias bibliográficas que se han ocupado sobre la distinción y catalogación de la obra de García Bacca en diferentes etapas. Aquí destacamos las que, a nuestro parecer, son las más importantes: Cf. Izuzquiza, 1984; BEORLEGUI, 1986; Beorlegui, 1988; Gimeno, 2015a.

4 «El sabio el shopós es un varón endemoniado»; «El filósofo es una manera de ser intermedia entre el sabio y el ignorante»; «El eros es necesariamente filósofo»; «El eros es hijo de Penía y Poros, del pobre rico en recursos».
} 
con un propósito claro, a saber: «De estas definiciones platónicas voy a deducir en qué consiste el tipo de endemoniamiento propio y exclusivo del filósofo» (García Bacca, 1949, p. 9.)

Pero antes de que el filósofo español defina el sentido que para él posee el endemoniamiento característico del filósofo, García Bacca introduce repentinamente un elemento nietzscheano en su argumento. Concretamente, el elemento introducido por García Bacca no es otro que el símil de la "maroma» que Nietzsche relata en su Zaratrusta. Allí dice el filósofo alemán al respecto: «El hombre es una cuerda tendida entre el animal y el súper hombre, -una cuerda sobre el abismo» (Nietzsche, 2003, p. 38).

Podemos, pues, empezar a establecer la génesis de la propuesta ontológica garcibacquiana de principios de los años cuarenta del pasado siglo: el hombre (filósofo) es un sujeto sostenido en permanente «equilibrio» entre dos extremos (Dios y Hombre). Dice el propio García Bacca respecto a esta idea: «Fijemos, pues, con Platón, los dos extremos de la maroma: uno, en lo divino; otro, en lo mortal. Sobre la maroma, tres tipos de seres se mantienen en equilibrio cinético: el demonio, el sabio y el filósofo» (García Bacca, 1940, p. 9).

En un primer momento pudiera parecer que en su gestación ontológica respecto a los diferentes estados en los que se encuentra el hombre, García Bacca únicamente hace uso de las definiciones dadas por Platón. Nada más lejos de la realidad. El hecho de que García Bacca haga referencia y uso del símil nietzscheano de la "maroma», no puede ser reducido únicamente a una suerte de anécdota o referencia realizada por García Bacca de soslayo. Existe, tal y como comentamos al principio del presente trabajo, una sintonía favorable por parte de García Bacca al sentido dionisíaco expresado por Nietzsche. En este sentido, el valor de lo dionisíaco como expresión de lo corporal, dialéctico y cambiante cala profundamente en García Bacca. Tanto es así, que para poder entender el potencial de la influencia nietzscheana en la gestación del argumento ontológico garcibacquiano, es preciso combinar el sentido platónico de Dáimôn (como endemoniado, es decir, como intermediario entre los dioses y los hombres), con el valor y potencial dialéctico de querer no solo alcanzar la figura absoluta de Dios como representación de perfección: «Dios es dios precisa y primariamente porque es la sustancia por antonomasia, el ser por excelencia, lo hecho y lo definitivo por eminencia» (García Bacca, 1940, p. 9); sino que, además, debe ser interpretado como el horizonte que siempre se sitúa más allá del hombre corporal y humano.

Veamos este asunto con mayor detenimiento y profundidad. Para poder comprender con mayor acierto este momento crucial de la gestación ontológica garcibacquiana, es preciso tener presente que para el filósofo español, ambos extremos (Dios y Hombre), son interpretados como los límites ontológicos sobre los que se fija la «maroma» sobre la que se desarrolla el equilibrio ontológico. Ambos límites beben, en mayor o menor medida, del sentido platónicocristiano clásico en la tradición filosófica occidental. Es decir, que García Bacca toma a Dios no estricta ni necesariamente como la figura cristiana del padre creador, pero sí como símil de la figura o Forma de lo absoluto, fijo, claro e 
incorruptible. Es decir, como eidos del lo puro: «Dios es el hecho definitivo y, por tanto, el ser; así, en unidad y en puridad» (García Bacca, 1940, p. 11). Lo cual no implica que el filósofo español defienda esta definición clásica y sustancialmente particular respecto a la figura de Dios. De lo que se trata es de ejemplificar de modo gráfico un extremo ontológico hacia el cual, el filósofo en su estado de endemoniado tiende y se mueve.

Si uno de los extremos es Dios, en lo que se refiere al otro extremo, es decir, el hombre, no deja de reflejar lo antagónico a la idea de absoluto: "Mortal, en cuanto el otro extremo de lo divino, significa algo mucho más profundo: mortal es lo des-hecho y lo deshacedero, lo in-definido, lo no-ser, lo in-subsistente» (García Bacca, 1940, p. 11). Es decir, que al igual que hiciera García Bacca con Dios para representar el extremo de lo absoluto, García Bacca explota el sentido prosaico del hombre como lo contrario. Es decir, como lo indefinido, lo informe, lo ausente de verdad en un sentido eidético. Bajo estos extremos que guardan una voluntaria semejanza con el sentido clásico, García Bacca inserta un gen nietzscheano para significar y dotar de movimiento al esquema ontológico clásico.

Con la inserción del símil de la «maroma» en el argumento garcibacquiano, el filósofo español es capaz de situar sobre ella los diferentes estados del filósofo: filósofo, sabio y endemoniado. De ese modo, logra uno de sus principales objetivos, a saber: establecer una dialéctica de ascenso desde la idea prosaica de filósofo, hasta el estado de endemoniado, pasando por el estado intermedio de sabio. Dice García Bacca al respecto: «El filósofo para sostenerse en la maroma tiene que avanzar hacia sabio, y el sabio hacia demonio. Primitivamente, el demonio ha comenzado por ser filósofo y por ser sabio; y por consiguiente, el auténtico filósofo y el genuino sabio están endemoniados» (García Bacca, 1940, p. 12).

Lo cierto es que todavía queda opaco el sentido que García Bacca atribuye al estado de endemoniado. No menos opaca queda la influencia nietzscheana en todo este asunto. Pues bien, para poder cerrar el argumento y terminar de aclarar esas lagunas argumentativas garcibacquianas, es preciso retomar una vez más la idea de lo dionisíaco como elemento catalizador de todo el argumento. Para García Bacca, el auténtico filósofo está endemoniado, es decir, está situado en una suerte de equilibrio entitativo y ontológico que lo hace tender tanto hacia el ser (Dios) como hacia el no-ser (hombre/animal). El filósofo, en estado de endemoniado como intermediario entre lo humano y lo divino, queda retratado como un ente que siempre trata de ir más allá de sí desde sí mismo. Es decir, que el filósofo trata de «transfinitar» los propios límites que lo determinan y definen como hombre en tanto que imperfecto, indefinido, e inconcluso. El papel nietzscheano en la gestación ontológica garcibacquiana en este punto queda, pues, definida no sólo por la idea de «equilibrio» sobre la maroma sino, también, como el modo en el que se desarrolla el propio movimiento dialéctico desde la idea absoluta y bella del filósofo, hasta un estatus demoníaco, corporal, transfinitante y dionisíaco que caracteriza el estatus de «autenticidad» filosófica. 
En cierto sentido y orden de discurso, es posible identificar en este punto del argumento, ciertas reminiscencias por parte de García Bacca, respecto al asunto de la inversión platónica propia de la filosofía nietzscheana y que, con cierta maestría, García Bacca pone al servicio sus intereses ontológicos. A nuestro parecer, García Bacca aplica en el seno de su argumentación, cierto grado de la inversión platónica. Lo hace para revitalizar un nuevo modo de proceder dialéctico referido a los diferentes estadios del hombre filósofo sobre la maroma. Dice García Bacca: «Pero la maroma tendida ente Dios y la Nada funambulan el filósofo, el sabio y el demonio; son los únicos que saben sostenerse sin caer en el abismo. Y los tres no son tres cosas atadas cada una en su punto, en un punto calculable y único; sino tres estadios, tres estaciones de un mismo movimiento» (García Bacca, 1940, p. 12).

En definitiva, García Bacca está vinculando dos autores (Platón y Nietzsche) que, desde el punto de vista de la filosofía clásica, quedan objetivamente distantes o, más bien, opuestos y enfrentados. Para salvar dicha oposición, García Bacca toma aquellos aspectos concretos de ambos autores que contribuyen a la gestación y formación de su propia comprensión ontológica del filósofo. Un filósofo que, en primer lugar, queda definido por su estado de endemoniado. Es decir, por su disposición como intermediario entre lo definido y absoluto por un lado y, por otro, entre lo indefinido, informe e incompleto. Ese estado de endemoniado propio de la filosofía platónica, García Bacca lo combina, en segundo lugar, con otro estado ontológico propio de la propuesta metafísica nietzscheana, a saber, con el símil de la maroma y la noción de equilibrio.

El hecho de que el filósofo se encuentre en equilibrio sobre una maroma determina, en opinión de García Bacca, el tipo de movimiento particular que define al filósofo. Un estado en equilibrio dinámico y dialéctico que lo hace moverse en equilibrio hacia ambos extremos, es decir, hacía el «ser» y el «noser»; hacia Dios y el hombre. El hecho de que el estado de partida esperado y definido sea el de endemoniado es, a todas luces, un nuevo estatus ontológico predefinido por García Bacca y que, en cualquier caso, no es sencillo de apresar en su totalidad.

Por otra parte, el hecho de que el filósofo se encuentre en equilibrio entre los extremos de ser y no-ser, así como el hecho de que el movimiento dialéctico no sea específicamente ni de ascenso o de descenso, coloca el nuevo estatus ontológico garcibacquiano entre la dialéctica de ascenso platónica (de lo corporal a la idea), y la inversión dionisíaca planteada por Nietzsche. En cualquier caso, el movimiento establecido por García Bacca es una combinación de ambos tipos de dialéctica tal y como estamos tratando de demostrar. No quiere decir, pues, que el hecho de equilibrio ontológico planteado por García Bacca defina un estatus de quietud ontológica. Lo que quiere decir es que dicho movimiento, a pesar de tender hacia la idea de Dios entendida como creación, en ningún caso puede evitar el hecho de retroceder hacia el no-ser y, lo que es más determinante aún, que en cualquier caso pueda alcanzarse en algún momento el extremo representado por Dios. Dice García Bacca al respecto: 
«¿Qué es pues y por fin, estar endemoniado o ser demonio? [...] Pretender y tender a ser y ser hijo de Dios por la gracia no sólo es pecado sin estado de gracia. Lo grave de esta limitación en el plan evolutivo y ascensional de las cosas consiste en que ni se puede n se debe pretender ser algo más que hijo adoptivo de Dios». (García Bacca, 1940, pp. 12-13)

Es decir, García Bacca está estableciendo el límite insuperable para el filósofo endemoniado, a saber, la idea absoluta y definida de ser interpretada como creación. Debemos conformarnos y asumir el estatus ontológico en el que sitúa García Bacca al filósofo, a saber: Un ser endemoniado y en equilibrio capaz de transfinitar aquellos límites que lo pretenden fijar en un estado fijo y absoluto. El filósofo endemoniado, por su condición de funambulista, tiene que mantener el equilibrio a través de un movimiento dialéctico entre el ser y no-ser para no caer en la nada o estado de cualquierismo ${ }^{5}$.

\section{UN NUEVO ESTATUS ONTOLÓGICO CAPAZ DE LA TRANSFINITUD}

En lo que sigue de Invitación a filosofar, García Bacca establece una conexión y discusión con Heidegger ${ }^{6}$ que, si bien es crucial para la comprensión de la gestación ontológica garcibacquiana, no guarda una relación directa con la posible influencia de Nietzsche en la obra del filósofo español. En lo que a nosotros respecta, lo que tratamos de exponer y analizar es la posible vinculación garcibacquiana con la obra de Nietzsche, así como el sentido de la colisión lateral entre ambos autores.

En este sentido, es crucial exponer la conexión existente entre la categoría garcibacquiana de la «transfinitud», y la categoría nietzscheana de la «voluntad de poder». La primera dificultad a la hora de exponer esta relación compleja y opaca estriba en que tanto Nietzsche, así como García Bacca, no mantienen ni establecen una definición fija y estable de sus respectivas categorías. Tanto en Nietzsche así como en García Bacca, es necesario interpretar ambas categorías desde un punto de vista hermenéutico, crítico e histórico, es decir, tratando de completar su sentido. Por esa razón, nuestro modo de proceder en este apartado será, siguiendo las posibilidades que nos proporciona la hermenéutica como debate abierto entre los autores y nosotros, una suerte de comparativa e interpretación de los sentidos ocultos de ambas categorías.

5 García Bacca identifica en su obra de 1939 Introducción al filosofar, cinco estados ontológicos en los que se puede encontrar el hombre. Baste para el propósito de nuestro argumento nombrar dichos estados: a).

6 Para mayor información sobre la relación entre García Bacca y Heidegger consultar: Cf. García Bacca, 1962; Gimeno. X, 2014; Vizcaino. E, 2012. 


\section{1. La Voluntad de poder}

Con el fin de no limitar la comprensión y sentido de la expresión nietzscheana de «voluntad de poder» al manido sentido de imposición de nuestra voluntad, creemos necesario tomar un enfoque completamente distinto. En concreto, nos referiremos a la voluntad de poder desde el punto de vista y enfoque planteado por Heidegger en su obra Nietzsche ${ }^{7}$. La decisión de tomar como referente interpretativo las lecciones de Heidegger responden a una cuestión capital, a saber, el hecho de que la interpretación heideggeriana de la voluntad de poder nietzscheana trata de establecer una hermenéutica de aquellos elementos constitutivos del acabamiento de la metafísica a manos de Nietzsche. Es decir, de lo que se trata es de centrar categorías nietzscheanas tales como la voluntad de poder desde un enfoque metafísico y ontológico.

Desde ese punto de partida argumentativo podemos decir, tal y como afirma Heidegger respecto a la voluntad de poder:

«Como carácter fundamental del ente en su totalidad Nietzsche reconoce y pone lo que denomina la "voluntad de poder". Con este concepto no sólo está delimitado qué es el ente en su ser. Este título de voluntad de poder, que se ha vuelto corriente de múltiples maneras desde Nietzsche, contiene para él la interpretación de la esencia del poder». (Heidegger, 2013, p. 553)

En primer lugar debemos explicar la relación establecida por Heidegger entre el qué-es y la voluntad de poder. El hecho de explicar esta relación establecida por Heidegger, nos proporciona el tono adecuado para sintonizar con la frecuencia metafísica que el autor alemán trata de imponer a la hora de interpretar esta categoría nietzscheana.

Partiendo de la premisa del tono metafísico, cuando Heidegger emplea la expresión qué-es, a nuestro parecer, no está haciendo otra cosa que referirse a la clásica distinción determinada por Aristóteles entre el qué-es y el que-es o, de otro modo, entre la esencia (forma) y la existencia ${ }^{8}$. El uso por parte de Heidegger de la relación entre el qué-es y que-es con la voluntad de poder responde a un objetivo esencial, a saber: tratar de establecer las bases argumentativas de la propuesta metafísica nietzscheana. Para poder comprender mejor el sentido de la interpretación heideggeriana de la voluntad de poder planteada por Nietzsche es necesario tener presente dos cuestiones esenciales.

En primer lugar, Heidegger trata de aplicar el chasis metafísico desarrollado en su obra Ser y tiempo para tratar de ajustar su interpretación metafísica de la obra de Nietzsche. Esto quiere decir lo siguiente: Heidegger aplica la diferencia entre Ser y Ente a la obra nietzscheana, con el fin de establecer una semejanza

7 Para este punto hemos apoyado nuestra argumentación en la interpretación que Heidegger realiza de Nietzsche y la "voluntad de poder» en sus lecciones durante 1939 compiladas en su obra Nietzsche, 2013. pp. 553 en adelante.

8 Para mayor información sobre la distinción entre ambos términos en Aristóteles consultar. Cfr. ARISTóteles. Met. Z. (4, 1030a, 17). 
entre la «voluntad de poder» y el qué-es. Es decir, para definir la voluntad de poder como el carácter fundamental del ente:

«La voluntad de poder dice qué es el ente, es decir, como qué ejerce poder (como poder) [...] El qué-es (voluntad de poder) no es un en sí, al que circunstancialmente le corresponda el que-es. El qué-es, en cuanto esencia, es la condición de la vitalidad de la vida (valor) y en este condicionamiento es, al mismo tiempo, el que-es propio y único de la viviente, es decir, aquí, del ente en su totalidad». (Heidegger, 2013, p. 534)

Es decir, que Heidegger, si bien es plenamente consciente de que Nietzsche no supo establecer la distinción entre Ser y Ente debido a su inclusión absoluta dentro del paradigma clásico de la metafísica, sí considera que es posible establecer un cierto grado de intuición ontológica de dicha cuestión en la obra de Nietzsche. Para ello, Heidegger atribuye a la voluntad de poder el valor de qué-es como esencia del ente en su totalidad. Con dicha relación, Heidegger está asentando las bases argumentativas para el segundo de los argumentos capitales para la comprensión de esta cuestión, a saber, la idea de que Nietzsche constituye el acabamiento de la metafísica.

En segundo lugar, debemos comprender que para Heidegger, Nietzsche constituye el acabamiento de la metafísica no como punto y final definitivo de su legitimidad. De lo que se trata, más bien, es de comprender que el paradigma clásico de la metafísica, cuyo origen es atribuido a Platón, ha sido «acabado» y "consumido». En primer lugar, Heidegger considera que Nietzsche consuma el paradigma metafísico clásico a través de un ejercicio ontológico basado en la inversión el platonismo. La inversión del platonismo en Nietzsche se determina por invertir las polaridades establecidas entre lo «suprasensible» y lo «sensible». Para Nietzsche, el fin del proceso ascendente dialéctico no pude consumarse en lo suprasensible y eidético. Nada más lejos de la realidad. El proceso dialéctico ascendente acaba y comienza con la «vida» que, como no podría ser de otro modo, nace y muere en lo «sensible» y «corporal».

Una vez invertido el platonismo, cabe preguntarse cómo y en qué disposición ontológica queda la comprensión del ser del ente por parte del Dasein, a partir de dicha reestructuración. Nadie pone hoy en duda el sentido que esconde tras de sí el ejercicio nietzscheano del nihilismo. Es decir, el ejercicio nihilista a través de la "transvaloración de todos los valores»" trata de asentar las bases de una revolución axiológica a través de la muerte de Dios. La muerte de Dios es, pues, una forma de vindicar el final o acabamiento de un paradigma metafísico que ha dominado Occidente durante más de veinticinco siglos. Pero para poder dotar de sentido al ejercicio nihilista plateado por Nietzsche es

9 Nos referimos, como no, en primer lugar a las referencias apuntadas por Nietzsche dadas en su obra La genealogía de la moral: "Abordaré esas cosas en otro contexto, más a fondo y con más dureza (bajo el título "Historia del nihilismo europeo"; remito para ello a una obra que estoy preparando: La voluntad de poder, ensayo de una transvaloración de todos los valores)»(NIETzSCHE, 2007, p. 201). 
preciso, en primer lugar, sostener un principio metafísico capaz de apuntalar argumentativamente el nihilismo. En este caso, dicho argumento de base es la voluntad de poder que, una vez sostenida la inversión del platonismo como efectiva y necesaria, es capaz de aportar una base ontológica suficientemente estable, como para poder argumentar metafísicamente desde la creencia de que lo «sensible» y lo «corporal» son las restauradas potencias que dotan de sentido a la «vida» y los «valores» del hombre.

La voluntad de poder, pues, no se debe comprender en este orden de discurso, como la imposición sin más de mis preferencias vitales. Nada más lejos de la realidad. La voluntad de poder responde, en definitiva, la identificación de una nueva esencialidad del ente en su totalidad y, al mismo tiempo, expresión fáctica del acabamiento metafísico a través de la inversión del platonismo. Como primera consecuencia de dicha inversión, el ente nietzscheano cuya esencia viene definida por la voluntad de poder, aplica todo el potencial vital, corporal y emocional que lo determina para acabar con Dios y, al mismo tiempo, redefinirse ontológicamente.

Es en este sentido en el que debe interpretarse el segundo punto aquí expuesto, es decir, aquél que tiene que ver con el acabamiento de la metafísica por parte de Nietzsche:

«El acabamiento de la metafísica, es decir al erigirse y consolidarse de la acabada carencia de sentido, no le queda, por tanto, más que la extrema entrega al final de la metafísica en la forma de la transvaloración de todos los valores. En efecto, el acabamiento nietzscheano de la metafísica es en primer lugar una inversión del platonismo (lo sensible se convierte en el mundo verdadero, lo suprasensible en el mundo aparente). Pero en la medida en que, al mismo tiempo, la idea platónica, en su forma moderna, se ha convertido en principio de la razón y éste en valor, la inversión del platonismo se convierte en transvaloración de todos los valores. En ella, el platonismo invertido se transforma en ciego endurecimiento y aplanamiento. Ahora sólo existe el plano único de la vida que se da a sí misma y por mor de sí misma el poder de sí misma». (Heidegger, 2013, p. 539)

A la luz de las palabras de Heidegger, se puede comprender mucho mejor el sentido de lo expuesto en este tercer punto respecto a la voluntad de poder. Para Heidegger, la voluntad de poder surge, en primer lugar, como resultado de la inversión platónica. Invertir el platonismo sitúa la vida en una posición ontológica privilegiada. A propósito de dicha posición de privilegio, Nietzsche redefine la gestación y creación de valores (sustento vital), a propósito de la voluntad que la vida posee por imponer su nuevo poder como director de orquesta.

\subsection{La transfinitación garcibacquiana}

Con el fin de poder completar el sentido de la relación y posterior distanciamiento entre Nietzsche y García Bacca, es preciso tratar de explicar qué es 
y qué sentido esconde la categoría garcibacquiana de la «transfinitud $»^{10}$. Para poder comprender mejor el sentido metafísico de la transfinitud garcibacquiana, nosotros vamos a tratar de proceder del mismo modo que Heidegger hiciera con Nietzsche. Es decir, aplicando la premisa ontológico que establece la diferencia entre el qué-es y el que-es. Partiendo de esta premisa, nosotros afirmamos que en la propuesta metafísica garcibacquiana, la transfinitud hace referencia a la esencia del ente. Es decir, según nuestra afirmación, la transfinitud garcibacquiana trata de dar respuesta a la pregunta por el qué-es del ente en términos heideggerianos.

Para poder comprender las razones por la que nosotros hemos establecido esta relación, es menester recordar el segundo punto del presente trabajo, es decir, aquél que trataba de establecer y definir las bases de la gestación ontológica de la propuesta metafísica garcibacquiana a través de la tensión entre dos extremos (Dios y el hombre). Partiendo de esta tensión es posible emparejar el extremo de Dios con la idea de infinitud. Del mismo modo, es posible relacionar el extremo del hombre con la idea de finitud. Así, entre infinito y finito, se sostiene un ente en particular (el filósofo endemoniado) que, en un equilibrio constante (como sinónimo de movimiento dialéctico), determina la esencia del ente en su totalidad. Si esto es así, para García Bacca el filósofo endemoniado se mueve de finito a infinito a través de un movimiento dialéctico esencial que se define a través de la transfinitud: «La filosofía no posee objetos propios, sino que todo objeto puede ser sometido a un proceso de transfinitación, al paso al límite infinito; y esta operación transfinitar, pasar al límite infinito en todos los órdenes, clases de objetos y propiedades, es filosofar» (García Bacca, 1940, p. 17).

Es decir, para García Bacca el hombre queda definido en su qué-es como un ente que sustentado entre dos extremos (infinito-finito), es capaz de moverse transfinitantemente desplazando los límites que en cada ocasión le salen al paso. La transfinitud, al menos en este momento de la propuesta garcibacquiana, trata de definir el qué-es del ente a través del establecimiento de una novedosa

10 El termino «Transfinitud» que a continuación presentamos, García Bacca lo emplea a partir de 1928. Dicho término lo recupera, tal y como él mismo reconoce en su obra Confesiones (p. 126-127), de la obra de Fränkel, Einleitung in die Mengenlehre de 1928. En esa obra descubre los «transfinitos» de G. Cantor que chocan directamente contra la concepción clásica que García Bacca tenía sobre los conceptos de «finito» e «infinito». El filósofo español confiesa en un momento: «Entre finito e infinito se da lo transfinito, perfectamente definido y por leyes propias determinadas» Cfr. García Bacca 2000, p. 126. El término de transfinitud, tomado y heredado de su $1^{\circ}$ etapa escolástico-lógica, supone un choque de fondo contra sus concepciones filosóficas. El descubrimiento de un concepto como el de transfinitud generará tal impacto filosófico para el propio García Bacca, que junto a conceptos tales como «transustanciación» y el «Nos», se mantendrán de modo permanente — no sin ciertas alteraciones- presentes a lo largo de su extensa producción filosófica. Cabe recordar en este punto, que el no abandono de estos conceptos no implica, tal y como se podrá comprobar a lo largo de nuestro trabajo, una variación o mutación en el sentido inicial que el filósofo español otorga a dichas categorías. En el caso que nos ocupa, nosotros intentamos dar cuenta tanto del origen mismo del término en su sentido original - teoría de conjuntos original de Cantor-, a sí como en su sentido garcibacquiano a lo largo de su prolija obra filosófica. 
categoría ontológica capaz de describir cómo el ente impone su voluntad de transfinitar. Es decir, la transfinitud, trata de definir el poder de la capacidad creativa e inventiva del hombre para desplazar todos y cada uno de los límites que se le aparecen en su movimiento perpetuo entre infinitud y finitud. Dice García Bacca:

«El auténtico hombre es una transfinitud que, por ser tal y no ser infinitud, tiene que tener en cada momento unos límites u otros que superar. Porque el hombre es esencialmente transfinito, y no infinito puede y tiene que tener unos u otros límites a superar, un alimento para que su transfinitud se alimente de él, lo absorba y pueda tender grado tras grado hacia la Infinidad, hacia Dios». (García Bacca, 1940, p. 18)

Por lo que a nosotros respecta, hemos tratado de definir en clave heideggeriana en qué consiste el qué-es del ente en su totalidad para García Bacca. Debido a la concepción metafísica garcibacquiana que define al hombre como un equilibrio entre infinitud y finitud; y dado que el hombre jamás puede lograr alcanzar la infinitud, es necesario introducir un nuevo elemento capaz de describir la esencial del ente en dicha situación de equilibrio. Hemos concluido, por lo tanto, que dicha categoría capaz de apuntar al qué-es del ente en dicha circunstancia de equilibrio ontológico es la transfinitud. Una potencialidad ontológica encargada de describir el movimiento de empuje y arrastre de los límites que el hombre encuentra en el camino. Un movimiento transfinitante que pone de manifiesto la voluntad del hombre por tratar de alcanzar el limite de lo infinito. Un límite insuperable y una circunstancia ontológica que lo constituye como ente transfinitante.

\subsection{La vinculación entre la voluntad de poder y la transfinitud}

Nuestra intención en este punto es la de tratar de exponer el sentido de la relación ontológica que se establece entre Nietzsche y García Bacca a través de los términos de voluntad de poder y transfinitación respectivamente. Con ello, trataremos de poner las bases argumentativas para poder comprender correctamente el último de los puntos del presente trabajo, a saber, aquél en el que se expone la colisión lateral entre ambos autores.

A la luz de todo lo expuesto, podemos afirmar lo siguiente: ambos autores están atravesados por una suerte de corriente dialéctica de orientación ascendente. Si bien el punto de partida de ambos procesos dialécticos no es exactamente el mismo (puesto que Nietzsche parte de una inversión del platonismo y la muerte de Dios y, por su parte, García Bacca de un equilibrio ontológico entre Dios y el hombre); aún así, decimos, ambos autores desarrollan una dialéctica de ascenso. Tratemos de expliquemos esta afirmación con mayor claridad.

Tanto la voluntad de poder nietzscheana, así como la transfinitud garcibacquiana, son dos categorías ontológicas que tratan de dar respuesta a la pregunta por el qué-es del ente. Dichas interpretaciones ontológicas inclinan la interpretación de la esencia del ente hacia la autoconciencia de sí como sujetos 
«creadores». En Nietzsche la creación viene determinada por el conjunto de valores nuevos creados desde el interior del hombre (filósofo) que, tras el ejercicio nihilista de matar a Dios, está capacitado para empoderarse de sí y de su facticidad ${ }^{11}$. En el caso de García Bacca, la creación viene determinada por el «mundo». El hombre (filósofo) como sujeto endemoniado está condenado a crear e inventar su mundo desde el material neutro que tiene a la mano, a saber, el «universo» $»^{12}$.

En ambos autores, el acto creativo e inventivo (ya sea de valores o de mundo), se gesta en el hombre de carne y hueso. Una necesidad y responsabilidad creativa que determina el tipo de movimiento dialéctico ascendente en ambos autores. Si bien Nietzsche niega a Dios como sinónimo de absoluto, fijo, universal y suprasensible con el fin de recuperar la facticidad del cuerpo como sinónimo de lo sensible, en García Bacca existe un inclinación relativamente semejante. Para García Bacca, el equilibrio ontológico entre Dios y Hombre es una manera de indicar el horizonte hacia el que debe dirigir su potencial creativo el hombre, a saber, la idea de absoluto entendido como creador.

La aparente aporía que parece existir entre el hecho de que Nietzsche niegue a Dios y que, por otro lado García Bacca tienda a Él no constituye, en este caso, una contradicción de ningún tipo. Si bien ambos extremos parecen oponerse, lo cierto es que ambos autores toman la figura de Dios en sentidos diferentes. Si pensamos en la idea de creación como sustento ontológico de ambas argumentaciones, cabe preguntarse si para Nietzsche, no es igualmente cierto que el epítome ideal de la creación viene determinado por Dios. Independientemente de que Nietzsche identifique al hombre como fuente de creación, lo cierto es que García Bacca hace exactamente lo mismo. Y lo hace porque en ambos autores, la figura de Dios está sustancialmente secularizada.

Es decir, tanto Nietzsche como García Bacca, toman la figura de Dios como sinónimos de lo absoluto, definido y universal. En el sentido de Nietzsche, lo absoluto se identifica con los valores y la regulación moral del hombre. En García Bacca, lo absoluto se identifica con la capacidad creadora e inventora. En ambos casos, pues, Dios debe ser interpretado como una forma de representar

11 Para mayor información sobre este fenómeno de empoderamiento ontológico como sujeto creador de valores, consultar Cf. Nietzsche, 2006.

12 La distinción garcibacquiana entre «Universo» y «Mundo» es clave para la comprensión de su propuesta metafísica. Resulta imposible debido a la evidente falta de espacio tratar con el rigor que merece esta distinción. Aún así, baste al lector con el siguiente apunte: la distinción entre Universo y Mundo la emplea García Bacca para diferenciar el estado «neutral» de las cosas en el «Universo» que, a través del potencial creativo e inventivo del hombre, va transformando y acomodando a sus necesidades a través del empleo de «enseres». El Universo neutro transformado a su imagen y semejanza a través de enseres es lo que García Bacca denomina «mundo». Son infinidad las referencias bibliográficas en las que García Bacca expone esta distinción. Nosotros apuntamos una por su importancia y relevancia, a pesar de existir otras muchas que deben ser tenidas en consideración. Cf. GARCía BACCA, 1963. 
el epítome de lo definitivo y absoluto ya sea como valor moral, ya sea como potencial creador e inventor de mundo o de valores.

Salvado el escollo argumentativo de la aparente aporía existente entre las ideas de Dios y de Hombre entre ambos autores, cabe preguntarse si, en el fondo, Nietzsche y García Bacca no defienden posiciones profundamente semejantes respecto al núcleo ontológico que sostiene sus argumentos, a saber, la capacidad creadora e inventora del hombre.

Si esto es así, tanto la voluntad de poder nietzscheana como la transfinitud garcibacquiana, son empleadas como categorías ontológicas que tratan de responder a la pregunta por el que-es del ente desde la premisa de la creación. Independientemente de crear valores o mundo, en ambos casos el responsable de dicha creación es el hombre (filósofo). Poco importa que la figura de Dios sea condenada o absuelta. Lo que importa es que en ambos casos la figura de Dios es secularizada y que, además, ambos autores convergen en la idea de focalizar el potencial creativo e inventivo del hombre, como respuesta a la pregunta por el qué-es del ente. Voluntad de poder y transfinitud mantienen una relación de parentesco tan opaca como cierta. Ambas posturas metafísicas son hijas legítimas de la creación e invención humana, es decir, de la inversión de un paradigma metafísico que respondía a la pregunta por el qué-es del ente desde la idea de Dios.

Destronada e invertida la dialéctica dominante, se abre un espacio para la reconquista y empoderamiento de la capacidad creativa e inventiva del hombre que, en su condición de condenado o endemoniado, debe afrontar su nueva condición ontológica que lo determina como un ser potencialmente creativo e inventivo del mundo que habita. Un mundo que desde los valores (Nietzsche) o desde los enseres (García Bacca), ahora se presenta como cambiante, dialéctico y sujeto a variaciones y trasformaciones.

\section{La colisión lateral entre Nietzsche y García Bacca}

Desentrañada y expuesta la compleja relación que mantiene la voluntad de poder nietzscheana y la transfinitud garcibacquiana, es hora de presentar los motivos de la colisión lateral que se produce entre ambos autores.

Partiendo de la premisa de que ambas propuestas convergen en la idea de la creación e invención como respuesta ontológica a la pregunta por el qué-es del ente (ya sea a través de la voluntad de poder, ya sea a través de la transfinitud), lo que cabe plantearse es lo siguiente: ¿ambas propuestas se desarrollan también de modo paralelo? Lo cierto es que no. La aproximación entre ambas propuestas es tan radical, que en un momento de su desarrollo las dos versiones ontológicas colisionan inevitablemente de modo lateral. Como si se tratara de dos bolas de billar, ambas propuestas ontológicas colisionan tomando trayectorias diferentes. 
En el caso de Nietzsche, la defensa de una creación de valores por parte del hombre termina por condensar y cristalizar todo su potencial en la figura del «superhombre»: "Yo os enseño el superhombre. El hombre es algo que debe ser superado. ¿Qué habéis hecho para superarlo?» (Nietzsche, 2003, p. 36). Para Nietzsche, el hombre tiene que conquistarse y empoderarse de sí y para sí mismo hasta el punto de ser superado. Por eso, Nietzsche trata de condensar en la idea del superhombre la representación del ejercicio desvalorizado, transvalorativo y nihilista que quiere para sí, y que ha definido como original respuesta a la pregunta por el qué-es del ente. El superhombre nietzscheano es la representación metafórica de un supuesto estado ontológico elevado por encima de la condición humana media. Trata de representar el horizonte axiológico deseado hacia el que todo hombre debe tender, si realmente quiere asumir y llevar a cabo la tarea y responsabilidad de crear un mundo a su imagen y semejanza.

Dentro de estos parámetros, nosotros nos preguntarnos si existe algún modo de regular o conciliar las distintas voluntades de poder creadas a partir del empoderamiento del hombre a propósito de la conquista de un nuevo estatus ontológico, a saber, el superhombre. Nos preguntamos si existiría modo o manera de conciliar dentro de una hipotética comunidad política de superhombres, las distintas tensiones producto de las diversas voluntades de poder. Nietzsche nos insta a empoderarnos de nosotros mismos a través de la autoconquista de nuestra capacidad creativa y nuevo nacimiento como superhombre, pero de lo que no habla es de cómo y de qué manera pueden conciliarse políticamente las distintas posiciones axiológicas de diferentes superhombres. Si el origen y fin de la creación de nuevos valores queda en manos de cada superhombre, entonces surge de si es posible conciliar las diversas voluntades de poder.

En el caso de García Bacca, el hecho de que la transfinitud se presente como respuesta tentativa a la pregunta por el qué-es del ente, sumado al hecho de que esta respuesta converja, al igual que en Nietzsche, en la capacidad creadora e inventora del hombre, no deviene, en ningún caso, en una suerte de solipsismo ontológico. García Bacca, a mediados de la década de los 60 introduce un nuevo ingrediente en la receta ontológica que, a nuestro juicio, es capaz de salvar su propuesta del solipsismo ${ }^{13}$. Tal y como presentamos en otro trabajo nuestro (Gimeno, 2015b), la introducción de la categoría del «Nos» por parte de García Bacca es, en definitiva, un esfuerzo por aunar el conjunto de transfinitudes (voluntades de poder) en el interior de su propuesta metafísica. Es decir, García Bacca descubre que su propuesta metafísica necesita del conjunto de transfinitudes, si realmente pretende lograr por parte de la humanidad, un nuevo modelo de humanismo aún por venir:

«Pueblo es invento. Pueblo es Nos en mundo de enseres, no en universo de cosas. No es suma de hombres en un universo de cosas que él: hombre y los

13 Sin duda alguna, la obra de referencia en la que García Bacca expone el sentido y valor del Nos es en: Invitación a filosofar según espíritu y letra de Antonio Machado, publicada en 1967. 
hombres no han creado o recreado a su imagen y semejanza. Pueblo es Todo de hombres que han reformado cosas que no veían ni miraban al hombre en cosas que los miren a todos como Todo; y transformando cosas que los veían, mas no los miraban a todos, en cosas que, además de verlos a Todos, los miren como Todo. Tal proceso es, en uno, creación del Pueblo y creador de Pueblo. El universo de los seres, humanizado por los inventos del hombre, es creador de Pueblo, quien se mueve, vive y es entre seres humanizados, cual en su mundo. A su vez: el hombre, en cuanto inventor es creador de mundo - transustanciando por milagro universo de seres en mundo de enseres. El Pueblo es creador de mundo.» (García Bacca, 1967, pp. 187-188)

Es decir, nosotros consideramos que García Bacca parece tener muy presente la posibilidad de que su proyecto dialéctico ascendente fracase, si dicho proyecto no es capaz de dar cuenta del resto de potenciales creativos e inventivos. No es casual que en su obra Existencialismo, García Bacca dedique un gran número de artículos a la obra de Heidegger tratando de criticar y apuntar el mismo defecto, a saber, el riesgo que corre una propuesta ontológica tendiente al solipsismo. Entre los riesgos de una dialéctica de este tipo, existe la posibilidad de dirigir la autoconciencia del hombre hacia un callejón sin salida, es decir, hacia un punto de no retorno donde la conciencia se descubre como limitada o abocada a la nada.

Para no encerrar ontológicamente al hombre en una ratonera solipsista, García Bacca integra la categoría del «Nos». Esta categoría compleja y difícilmente definible en una frase, nace como consecuencia de las relecturas por parte de García Bacca de la obra de Machado y Marx. El filósofo español parece advertir que ninguna propuesta ontológica que no cubra la justificación argumentativa de la facticidad social es, en el mejor de los casos, insuficiente y poco ajustada a la realidad humana.

No basta, pues, con proponer un metafísica de la creación y la invención, es decir, del empoderamiento de sí como Dios creador de mundo. Además, es necesario que la potencialidad creadora e inventora del hombre desde su estado de "persona», converja con otros hombres en estados ontológicos idénticos y, por qué no, diferentes. García Bacca cree que si el hombre debe encaminar su existencia hacia un humanismo aún por venir, futuro y en construcción, ese humanismo no puede estar formado por individualidades. Nada más lejos de la realidad, el futuro y porvenir de la sociedad está en manos de la convivencia del conjunto de hombres que, desde su posición y empoderamiento como hombres transfinitantes, sean capaces de consolidar un comunidad ( Nos») de hombres creadores e inventores de mundo.

\section{CONCLUSIÓN}

En presente trabajo hemos tratado de presentar la compleja relación que existe entre Nietzsche y García Bacca desde un punto de vista metafísico. Hasta donde nosotros tenemos constancia, no existe ningún trabajo que haya tratado 
de explicar en profundidad un asunto tan complejo como necesario en la cartografía y estudio de la obra de García Bacca. Creemos que era necesario tratar de cubrir un vacío dentro de los estudios de la vida y obra del filósofo español y, en mayor medida, un asunto que tanto ha pasado desapercibido como el aquí presentado.

Es cierto que la relación entre Nietzsche y García Bacca se presenta como difícil, enrevesada y opaca en muchos de sus aspectos. Pero de lo que no nos cabe la menor duda, es que la relación metafísica entre Nietzsche y García Bacca es crucial para poder comprender e interpretar el potencial de la propuesta metafísica del filósofo español. En este trabajo, pues, hemos tratado de arrojar luz sobre la semejanza que existe entre Nietzsche y García Bacca a través del análisis de dos categorías ontológicas como son la voluntad de poder y la transfinitud. Hemos tratado de demostrar cómo García Bacca hereda explícitamente algún elemento argumentativo de la propuesta nietzscheana. Hemos analizado el modo en el que García Bacca combina de un modo tan heterodoxo pero eficiente, elementos de la propuesta platónica por un lado, y de la interpretación ontológica nietzscheana por otro lado. También hemos analizado y comparado el potencial dialéctico de las categorías de voluntad de poder y la transfinitud, para tratar de argumentar cómo y en qué medida, ambas categorías son empleadas como herramientas para tratar de dar una respuesta a la pregunta por el qué-es del ente, e interpretada en clave de creación.

Finalmente, hemos tratado de explicar cómo García Bacca procura salvar el peligro del solipsismo ontológico, a través de la categoría del «Nos». La inserción por parte de García Bacca de la categoría del «Nos» es, a nuestro modo de parecer, el salvoconducto que la propuesta metafísica garcibacquiana emplea para sostener argumentativamente su tesis del humanismo positivo aún en construcción y todavía por venir.

\section{BiBLIOGRAFÍA}

García Bacca. J. D. (1940). Invitación a filosofar. Vol I: La forma del conocer filosófico. México: Fondo de Cultura Económica.

- (1947/1990). Nueve grandes filósofos contemporáneos y sus temas. Vol I: Bergson, Husserl, Unamuno, Heidegger, Scheler, Hartman. Vol II: W. James, Ortega y Gasset, Whitehead. Caracas: Imprenta Nacional, Ministerio de Educación de Venezuela, 1947. Nueva edición en 1990. Anthropos.

- (1962). Existencialismo. Xalapa (México): Universidad Veracruzana.

- (1963). Metafísica natural estabilizada y problemática metafísica espontánea. México: Fondo de Cultura Económica.

- (1967). Invitación a filosofar según espíritu y letra de Antonio Machado. Barcelona: Anthropos.

- (2000). Confesiones. Autobiografía intima y exterior. Barcelona: Anthropos.

Aristóteles (1994). Metafísica. Madrid: Gredos.

Beorlegui, C. (1986). La filosofía del hombre en J.D. García Bacca. Tesis doctoral. 2 vol. Bilbao. 
- (1988). La audacia de un pensar. Bilbao: Universidad de Deusto.

Gimeno. X. (2015a). Juan David García Bacca: una invitación a la facticidad de la «transfinitud» y la «transustanciación». La necesidad social del «Nos»y del método dialéctico de transustanciación. Tesis doctoral, Valencia.

- (2015b). «La categoría de Nos en Juan David García Bacca y la importancia de Antonio Machado», en: Revista de estudios filosóficos, Valladolid.

- (2014). «El ataque de Juan David García Bacca a la categoría de Sorge heideggeriana», en: Estudios fenomenológicos, Vol. 11, Madrid.

Heidegger, M. (2009). Ser y tiempo. Madrid: Trotta.

- (2013). Nietzsche. Barcelona: Ariel.

Izuzquiza, I. (1984). El proyecto filosófico de Juan David García Bacca. Barcelona: Anthropos.

Nietzsche, F. (2003). Así habló Zaratustra. Madrid: Alianza Editorial.

- (2006). La voluntad de poder. Madrid: Alianza Editorial.

- (2007). La genealogía de la moral. Madrid: Alianza Editorial.

- (2012). El nacimiento de la tragedia. Madrid: Alianza Editorial.

Platón (2004). El banquete. Introducción de Carlos García Gual; traducción y notas de Fernando García Romero. Madrid: Alianza Editorial.

Vizcaíno, H. (2012). «García Bacca, lector crítico de Heidegger 2». Y en: Revista filosofía Eikesia Julio de 2012, pp. 147-172.

Universidad de Valencia

XAVIER Gimeno Monfort

Facultad de filosofía y CC.EE

Departamento de filosofía del derecho, moral y política

xagimon@hotmail.com

[Artículo aprobado para publicación en diciembre de 2016] 